\title{
Prediction in Mental Health Research and Its Limits (or why life can only be understood backwards but must be lived forwards)
}

\author{
Nelson, B. ${ }^{1,2}$ * \\ Hartmann, J.A. ${ }^{1,2}$
}

\section{EIP Editorial}

This is the author manuscript accepted for publication and has undergone full peer review but has not been through the copyediting, typesetting, pagination and proofreading process, which may lead to differences between this version and the Version of Record. Please cite this article as doi: 10.1111/eip.12530

This article is protected by copyright. All rights reserved. 
${ }^{1}$ Orygen, The National Centre of Excellence in Youth Mental Health, Melbourne, Australia

${ }^{2}$ Centre for Youth Mental Health, University of Melbourne, Australia

* Corresponding author. Contact e-mail: Barnaby.Nelson@orygen.org.au

There is much focus in the mental health field on prediction research, as reflected in the papers published in this journal. Longitudinal prediction of disorder onset (with "transition" to psychotic disorder being the paradigmatic example) has been the main focus, but research has also addressed outcomes such as psychosocial functioning, recovery, and treatment response, defined both in clinical and biological terms. While efforts are continually made to improve our predictive models through advancing analytical strategies (e.g., the current trend towards machine learning) or grounding predictions in our evolving understanding of pathoaetiological mechanisms of disorder onset and trajectory, the question of the constraining or limiting factors on prediction is rarely raised.

This question might be approached in a number of ways. Here we provide some reflections on limitations of current approaches and then discuss some possible inherent limitations in psychiatric prediction research.

\section{Static versus dynamic approaches to prediction}

Psychiatric prediction research has shown an overreliance on one-off sampling of cross- 
sectional data (i.e., a "snapshot" of clinical state and other risk markers). Predictive models, even if multi-modal in nature ${ }^{1}$, are limited to a single point in time (study entry or clinic presentation). We have recently argued that predictive modeling may benefit from moving from this convenient but rather static approach to taking dynamic, timedependent changes into account ${ }^{2}$. While this point has been made with regards to psychosis $^{2-4}$ and suicide ${ }^{5}$ research, it applies across the field of prediction. Crossdisciplinary approaches to complex system structures and changes (e.g., as seen in ecology or economics), such as dynamical systems theory, network theory, instability mechanisms, chaos theory and catastrophe theory, offer useful models that can be applied to the emergence (or decline) of psychopathology. However, there are also theoretical models within mental health research, such as fluid vulnerability theory ${ }^{6}$, that can provide a framework for understanding the predictive value of longitudinal interaction between risk factors. This type of predictive modelling requires repeat longitudinal assessment of relevant variables through either, or a combination of, micro- (momentary, day-to-day) and macro- (months, years) level assessments. Such an approach to prediction research may more accurately model the highly dynamic nature of psychopathology and system change $^{7}$, as well as have treatment implications, such as introducing a means of identifying early warning signs for mental state deterioration or improvement, as well as critical periods of risk. Empirical work adopting this dynamic approach has already shown significant promise ${ }^{4,8-10}$ and may be particularly relevant to individual-level, 
“precision" medicine/prediction ${ }^{8}$.

\section{Broad outcomes}

The outcome of interest in prediction research is often a broad category. In the case of psychosis prediction research, the main outcome of interest, first episode of psychosis (FEP), is broad and varied in clinical presentation (a multifarious phenotype $)^{11,12}$, as well as being defined in dichotomous terms ${ }^{13}$. From a service provision perspective this has been an important advance. However, as a single category of interest in prediction research it may present a constraining factor on predictive models. This is because there may be multiple underlying disease processes (with a complex mix of biological, psychological and social components) in the onset of the single category of FEP, reflected in part in the variety of FEP phenotypes. While these disease processes may all flow into a single common final pathway, such as dopamine dysregulation (on a neurochemical level) ${ }^{14}$ and threshold positive psychotic symptoms (on a clinical level), predictive models ideally need to capture the range of factors at play before this final common pathway. It is unlikely that a single model will be able to achieve this with a high degree of accuracy because of the multiple aetiological pathways in operation. To use an analogy, the category 'fruit' (=FEP) consists of the fruit sub-type 'banana' and the fruit sub-type 'orange' (along with a range of other fruit!), and these fruit sub-types have quite distinct properties from each other (=likely to have distinct aetiological pathways 
and associated predictive models). By widening the outcome of interest to the broad category of 'fruit' these important distinctions will not be detected and the predictive power of a single model will be constrained. Similar points might be made about other areas of mental health prediction research, such as suicide risk or severe depression ${ }^{15}$. A more powerful approach might be to map the variety of underlying disease processes (across psychological, social, neurocognitive, neurobiological, genetic domains) on to the range of phenotypes within the broad outcome categories ${ }^{16}$ in order to generate and test more specific and targeted predictive models and clinical identification strategies.

\section{Natural history of disorder versus treatment response}

Most prediction studies in the mental health field use patient samples who are receiving treatment of some kind, even if this is "treatment as usual" in the context of an intervention trial or a longitudinal observational study. However, the language of most of these studies remains based on trying to uncover the "natural history" of disorder, e.g. neurostructural abnormalities that characterise "ultra high risk" patients who progress to psychotic disorder. However, the provision of treatment confounds the researcher's window into natural disease progression. We are not proposing that treatment should not be provided in such prediction studies, but rather that we need to better incorporate the role of treatment into the semantics and concepts of prediction research. For example, "variables that predict reduced treatment response" might be a more accurate way of 
characterising many variables identified in predictive models rather than "predictors of illness progression". It is possible that different variables might predict illness progression in the context of no treatment (natural history of disorder) compared to the context of (psychological or pharmacological) treatment provision, whether this is controlled trial treatment or standard care. This could possibly be tested in a longitudinal comparison of two samples with the same clinical phenotype, one of which is helpseeking/receiving treatment and the other which is not, although this approach also has its limitations ${ }^{17}$.

In addition to these limitations to the approach taken in current prediction research, there may also be some inherent or 'built-in' limitations to psychopathology prediction research that are not so present, or at least not to the same degree, in other disciplines.

\section{Constraints on experimental manipulation and measurement}

To use an example from ecology, the resilience of a system (e.g., a lake) can be tested by measuring the time it takes to return to its previous state after it has been (experimentally or naturally) perturbed. Both perturbation and the lake's response can be characterised well and monitored closely. In psychiatric prediction research, we are limited in the way we can experimentally induce a perturbation or characterise a naturally occurring stressor in a person's life, and, importantly, we are limited in how often and what we can measure. 
Although simulation models ${ }^{18}$ and passive sensing ${ }^{19}$ may be ways of addressing these limitations, they do not promise to overcome this characteristic limitation entirely.

\section{Lack of precision and high probability of stochastic and chaotic factors}

Only few disciplines can achieve very high levels of predictive accuracy, when "prediction" is understood as using current parameters and variables to identify the likelihood of an event occurring at a future point in time ${ }^{20,21}$. Mathematics and physics enjoy a high level of precision in being able define relevant parameters/variables and often have high levels of control over factors that have been bearing on the model being examined. However, this is often not the case in mental health research. Firstly, it is very difficult for mental health researchers to define and quantify with high degrees of precision the variables of interest (mental states, biopsychosocial variables, etc.). Secondly, the factors that influence mental states (both within an individual and contextual factors) are broad and varied and unpredictable in themselves. On the psychosocial level, consider the mercurial and capricious nature of life events and relationships; on the brain level, the degree of complexity is vast and poorly understood. This possible inherent limitation in psychiatric research and a variety of social sciences was recognised as early as the 1920s in the economist Frank Knight's distinction between "risk", a quantifiable identity, and "uncertainty", referring to constructs which were not amenable to precise measurement or quantification ${ }^{22}$. 
Even in disciplines that enjoy high levels of precision there are limits to predictive capacity. In physics, quantum mechanics reveals difficulties in predicting some physical systems. In astronomy, when the movements of three objects are considered (e.g., the sun, earth and moon, sometimes referred to as the "three body" problem) the challenge of prediction over the longer term exponentially increases given the contribution of "chaotic" factors (slight uncertainties in the location, speed of movements, etc. of the planets that amplify over time, popularly referred to as the "butterfly effect"). The "predictability horizon" ${ }^{20}$ recedes when a system has a high degree of chaos/stochasticity built into it. Another illustrative example is weather patterns. Weather prediction is generally quite accurate for several days ahead (Melbourne excluded), but becomes nearly impossible for 3-4 weeks ahead. This is not due to problems in mathematics, weather modeling or computing, but it is the stochastic nature of the phenomenon/system itself (in this case, weather patterns emerging from states of the atmosphere and oceans). Psychopathology is likely to present the same inherent difficulties of a high degree of random and chaotic factors in its component features and in the biopsychosocial variables that impact it.

\section{High sensitivity to contextual factors?}

It is possible that a characteristic feature of psychopathology is high sensitivity to contextual factors and perturbations, possibly particularly during the developmental 
period of adolescence and early adulthood. An open question is to what degree the "system" of mental state is highly sensitive and reactive to such events compared to other systems, e.g. ecological systems and financial markets ${ }^{2}$. According to network theory the degree of sensitivity/resilience might depend on the level of interconnectedness and similarity/divergence of the system's component parts (its "architecture") ${ }^{23}$. The constitutive architecture of mental health/disorder is poorly understood at present, but if indeed it is a system that is highly sensitive and reactive to perturbations, and biopsychosocial perturbations are by their very nature highly unpredictable and stochastic (see above), then this places a limit on predictive modeling.

\section{Humans react to predictions}

Solar eclipses and weather patterns pay no heed to predictions made about them. However, in contrast to physical objects and systems, humans have free will and can attempt to influence a predicted trajectory by manipulating the variables at play. While this may not be possible for certain medical conditions (e.g., inherited genetic diseases such as Huntington's disease), the level of risk for onset or course of mental disorder is likely to be amenable to change (e.g., by identifying triggering psychosocial contexts, reducing drug use, changing sleep-wake cycles and activity patterns, etc.).

\section{Psychopathology does not consist of cyclical repetition}


The strongest predictive models can be achieved for cyclical, repeated events. Some features of the natural world, such as the movement of ocean tides, planetary eclipses and seasons, fit this bill well. However, rare, non-cyclical events ("black swan" events ${ }^{21}$ ) are not as easily predicted. Mental states and factors that impact on mental states are not cyclical or repetitious in this strict sense but instead unfold over time, evolving and changing in the same way that a physical body evolves and changes over a lifespan. Again, this inherent feature constrains predictive models.

\section{Conclusion}

The above observations point to the fact that in mental health research we need to improve descriptive characterisation of clinical phenomena (the "object" of psychiatry ${ }^{24}$ ) and their correlates at different (neuro-, bio-, geno-) levels of analysis, with prediction being dependent on precise definition of variables/systems of interest. This is partly what has prompted warnings about the "death of phenomenology" in psychiatric research and

clinical practice ${ }^{24-26}$. A different reaction, although possibly borne of similar frustration, can be seen in the NIMH's move away, guided by the Research Domain Criteria (RDoC), from the DSM phenotype-based classification system and its attempt to base psychiatric nosology on neuroscience and behavioural science instead of descriptive phenomenology ${ }^{16,27}$. Secondly, there is a need for improved recognition and understanding of the contribution of dynamic and stochastic factors in mental health 
research. These factors will probably never be able to be fully measured, explained or accounted for (by their very nature) but the degree to which they contribute to psychiatric outcomes can be better modeled.

Inherent or "built in" limitations to psychiatric prediction does not at all mean that we should abandon predictive modeling. Prediction does not need to be perfect to be useful. Our models will of course remain probabilistic affairs, even if many of the issues flagged above are somehow addressed. The goal is to achieve a degree of predictive accuracy that is useful for clinical decision-making and to assist with understanding disease processes. This will probably not be achieved by "number crunching" with ever greater technical sophistication, but also by critically considering the concepts at play in psychiatric prediction, with one eye on what is going on in other disciplines. 


\section{References}

1. Nieman DH, Ruhrmann S, Dragt S, Soen F, van Tricht MJ, Koelman JH, Bour LJ, Velthorst E, Becker HE, Weiser M, Linszen DH, de Haan L. Psychosis prediction: stratification of risk estimation with information-processing and premorbid functioning variables. Schizophr Bull. 2014;40(6):1482-1490.

2. Nelson B, McGorry PD, Wichers M, Wigman JTW, Hartmann JA. Moving From Static to Dynamic Models of the Onset of Mental Disorder: A Review. JAMA Psychiatry. 2017;74(5):528-534.

3. Yuen HP, Mackinnon A. Performance of joint modelling of time-to-event data with time-dependent predictors: an assessment based on transition to psychosis data PeerJ. 2016:4:e2582.

4. Yuen HP, Mackinnon A, Nelson B. A method for analysing transition to psychosis: joint modelling of time-to-event outcome with time-dependent predictors. International Journal of Methods in Psychiatric Research. 2017; In press. DOI 10.1002/mpr.1588.

5. Bryan CJ, Rudd MD. The Importance of Temporal Dynamics in the Transition From Suicidal Thought to Behavior. Clin Psychol Sci Prac. 2016;23:21-25.

6. Rudd MD. Fluid Vulnerability Theory: A cognitive approach to understanding the process of acute and chronic suicide risk. In: Ellis TE, ed. Cognition and suicide: 
Theory, research, and therapy Washington, DC: American Psychological Association; 2006:355-368.

7. McGorry P, Nelson B. Why We Need a Transdiagnostic Staging Approach to Emerging Psychopathology, Early Diagnosis, and Treatment. JAMA Psychiatry. 2016:1-2.

8. Wichers M, Groot PC, Psychosystems ESMGEWSG. Critical Slowing Down as a Personalized Early Warning Signal for Depression. Psychother Psychosom. 2016;85(2):114-116.

9. Wichers M, Wigman JTW, Myin-Germeys I. Micro-Level Affect Dynamics in Psychopathology Viewed From Complex Dynamical System Theory. Emotion Review. 2015;7(4):362-367.

10. van de Leemput IA, Wichers M, Cramer AO, Borsboom D, Tuerlinckx F, Kuppens P, van Nes EH, Viechtbauer W, Giltay EJ, Aggen SH, Derom C, Jacobs N, Kendler KS, van der Maas HL, Neale MC, Peeters F, Thiery E, Zachar P, Scheffer M. Critical slowing down as early warning for the onset and termination of depression. Proc Natl Acad Sci U S A. 2014;111(1):87-92.

11. Nelson B, Yung AR. Psychotic-like experiences as overdetermined phenomena: When do they increase risk for psychotic disorder? Schizophr Res. 2009;108(13):303-304.

This article is protected by copyright. All rights reserved. 
12. Nelson B, Thompson A, Yung AR. Not all first-episode psychosis is the same: preliminary evidence of greater basic self-disturbance in schizophrenia spectrum cases. Early intervention in psychiatry. 2013;7(2):200-204.

13. Yung AR, Nelson B, Thompson A, Wood SJ. The psychosis threshold in Ultra High Risk (prodromal) research: is it valid? Schizophrenia research. 2010;120(1$3): 1-6$.

14. Howes OD, Kapur S. The dopamine hypothesis of schizophrenia: version III--the final common pathway. Schizophr Bull. 2009;35(3):549-562.

15. Fried EI, Nesse RM. Depression sum-scores don't add up: why analyzing specific depression symptoms is essential. BMC Medicine. 2015;13(1):72.

16. Nelson B, Hartmann JA, Parnas J. Detail, dynamics and depth: Useful correcttives for some current research trends. Under review. 2017.

17. Fusar-Poli P, Rutigliano G, Stahl D, Schmidt A, Ramella-Cravaro V, Hitesh S, McGuire P. Deconstructing Pretest Risk Enrichment to Optimize Prediction of Psychosis in Individuals at Clinical High Risk. JAMA Psychiatry. 2016;73(12):1260-1267.

18. Borsboom D, Cramer AO. Network analysis: an integrative approach to the structure of psychopathology. Annu Rev Clin Psychol. 2013;9:91-121.

19. Insel TR. Digital phenotyping: Technology for a new science of behavior. JAMA. 2017.

This article is protected by copyright. All rights reserved. 
20. Silver N. The signal and the noise: Why so many predictions fail - but some don't. New York: Penguin; 2012.

21. Taleb NN. The black swan: The impact of the highly improbable. New York: Random House; 2007.

22. Knight FH. Risk, uncertainty, and profit. Boston, MA: Hart, Schaffner \& Marx; 1921.

23. Borsboom D. A network theory of mental disorders. World Psychiatry. 2017;16(1):5-13.

24. Parnas J. The RDoC program: psychiatry without psyche? World Psychiatry. 2014;13(1):46-47.

25. Andreasen NC. DSM and the death of phenomenology in america: an example of unintended consequences. Schizophr Bull. 2007;33(1):108-112.

26. Parnas J, Bovet P. Psychiatry made easy: operation(al)ism and some of its consequences. In: Kendler K, Parnas J, eds. Philosophical Issues in Psychiatry III: The Nature and Sources of Historical Change. Oxford: Oxford University Press; 2017:191-212.

27. Cuthbert BN. The RDoC framework: facilitating transition from ICD/DSM to dimensional approaches that integrate neuroscience and psychopathology. World Psychiatry. 2014;13(1):28-35. 


\section{University Library}

\section{- M M N E R VA A gateway to Melbourne's research publications}

Minerva Access is the Institutional Repository of The University of Melbourne

Author/s:

Nelson, B;Hartmann, JA

Title:

Prediction in mental health research and its limits (or why life can only be understood backwards but must be lived forwards)

\section{Date:}

2018-10-01

\section{Citation:}

Nelson, B. \& Hartmann, J. A. (2018). Prediction in mental health research and its limits (or why life can only be understood backwards but must be lived forwards). EARLY INTERVENTION IN PSYCHIATRY, 12 (5), pp.767-770. https://doi.org/10.1111/eip.12530.

Persistent Link:

http://hdl.handle.net/11343/294070 